\title{
Les femmes de la Salpêtrière: trois siècles d'histoire hospitalière parisienne
}

Dora B. Weiner

A dix-sept ans, le roi Louis XIV avait de graves problèmes à résoudre. Les rues de Paris pulullaient de mendiants sans abri, de sorte que les honnêtes bourgeois ne se sentaient plus en sécurité; et des vagabonds, chassés de chez eux par les guerres et la misère, infestaient les routes de l'Ile de France. Heureusement que la mère du roi, la princesse espagnole Anne d'Autriche et son intime conseiller, le cardinal Mazarin, connaissaient le chemin du salut pour tous ces sans-abri: les enfermer, les faire travailler, leur imposer les devoirs de la religion. Tels furent les débuts de l'Hôpital général, surveillé par le Parlement de Paris et le Lieutenant général de police, administré par une douzaine de grands bourgeois, financé par le roi et des personnes pieuses parmi lesquelles Madame de Miramiones et la duchesse d'Aiguillon, nièce de feu le cardinal de Richelieu. ${ }^{1}$

Dès le début, en 1656, l'Hôpital général se divisait en quatre institutions: la Pitié pour l'administration,l'Hôtel de Scipion pour le ravitaillement, l'Hospice de Bicêtre pour les hommes, l'Hospice de la Salpêtrière pour les femmes. Ces hospices ne prévoyaient point de soins médicaux, bien que beaucoup de ces pauvres fussent en mauvaise santé, souffrant souvent de maladies chroniques.

Le Roi Soleil, qui allait bientôt se retirer dans son magnifique château de Versailles, voulait montrer au monde qu'il logerait ses pauvres aussi somptueusement que ses invalides. La construction des nouveaux bâtiments de la Salpêtrière fut donc confiée à Louis Le Vau (1612-1670), l'architecte de l'église St. Louis de Versailles, suivi par son élève Libéral Bruant (1635-1697). Ils

1 Poujol, Robert, «La naissance de l'Hôpital général de Paris d'après des documents inédits (papiers Minachon),» La Salpêtrière hier et aujourd'hui, Numéro spécial de L'Hôpital à Paris. Août 1982,11-34; voir 15-16.

Dr. Dora B. Weiner, 12-138 Health Sciences Center, UCLA School of Medicine, Los Angeles, CA 90024, U.S.A. 


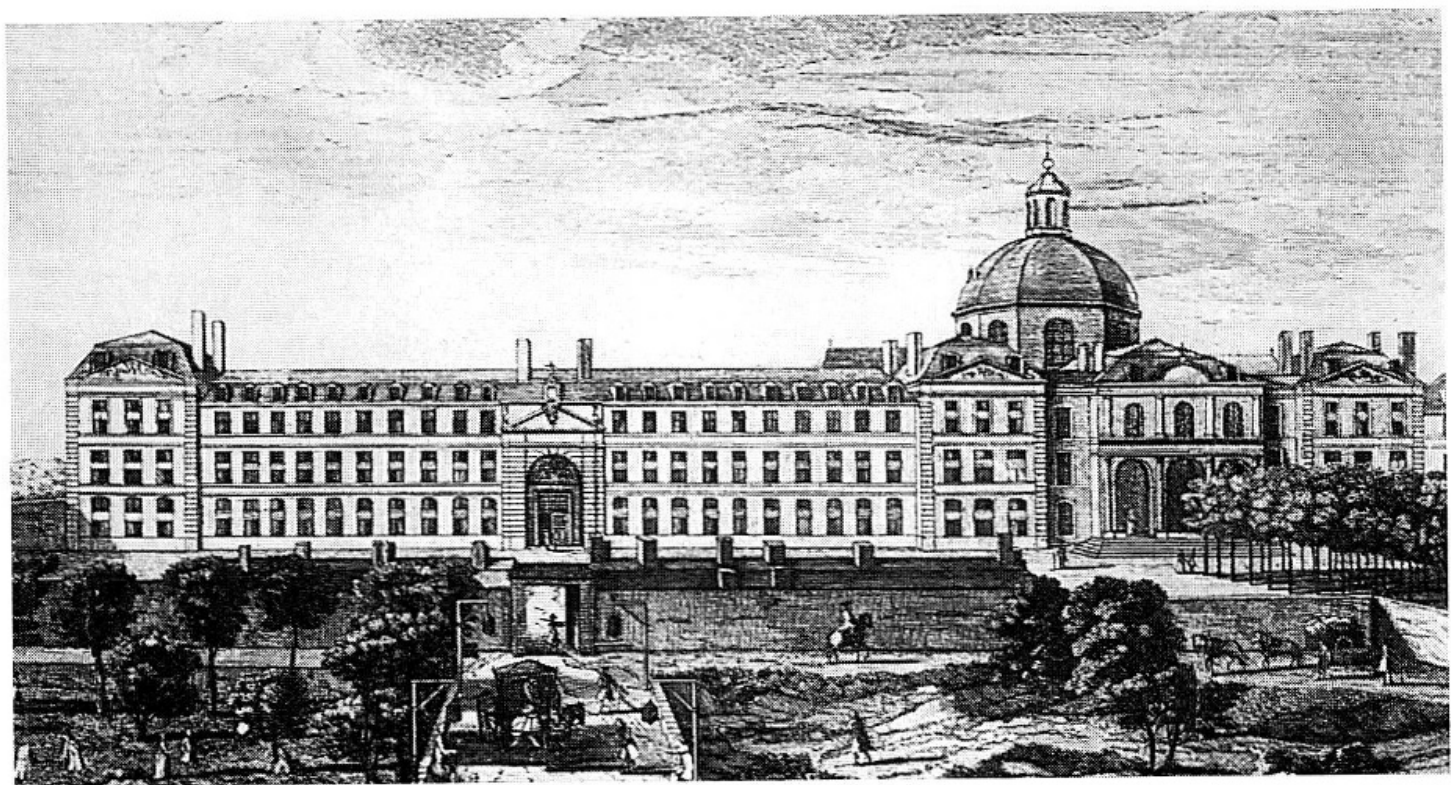

Fig. 1. Vue de l'Hospice de la Salpêtrière au $17^{\mathrm{e}}$ siècle par J. Rigaud.

construisirent la belle église St. Louis de la Salpêtrière remarquable par huit ailes rayonnant de l'autel central. Huit groupes de femmes pouvaient ainsi assister à la même messe sans s'entrevoir. Jules Hardouin-Mansart (1646-1708) ajouta le Dôme et tout fut terminé en 1678. « ... deux corps de logis,» lisonsnous, [dont l'aile Mazarin actuelle] «où sont logés les ecclésiastiques et officiers principaux et où sont les offices, cuisines, et ... quinze grands dortoirs [de 60 à 80 mètres] ... occupés par 628 femmes.» ${ }^{2}$ Ces 628 femmes de 1656 deviendront 3,000 à la fin du grand siècle et 8,000 cent ans plus tard. ${ }^{3}$

D'où venaient-elles en si grand nombre, ces femmes de la Salpêtrière? Le règlement y offrait un refuge aux bons pauvres, c.à.d. aux femmes de 60 ans de la région parisienne, valides mais sans moyens, et même à un petit nombre de couples âgés et indigents, une centaine à la fin du grand siècle, car le cardinal Mazarin avait fondé une section pour ménages. Ces pauvres étaient admis par le bureau qui siègeait à la Pitié deux fois par semaine. Ainsi, tous les mois, vers la fin de l'ancien régime, entre 150 et 300 femmes entraient à la Salpêtrière. Un quart étaient très vieilles, âgées parfois de plus de 70 ans, car le bureau les avait fait attendre faute de place. Elles venaient à la Salpêtrière pour y mourir, attirées par l'assurance d'un gîte, de nourriture et d'un minimum d'attention pendant leur agonie. Elles épargneraient ainsi à leur famille les frais d'un enterrement. Parmi les femmes âgées accueillies en Ven-

2 Puzin, Daniel et René César, «L'évolution historique du plan masse de la Salpêtrière.» $L a$ Salpêtrière hier et aujourd'hui, Numéro spécial de L'Hôpital à Paris, Août 1982, 35-42.

3 Benassis, Dr. «Hospice de la Salpêtrière,».in «Promenades médicales,» Revue thérapeutique, 1936, 4: 104-113,136-144,168-177, 199-208; le nombre de 3,000 femmes est cité la page 113. 


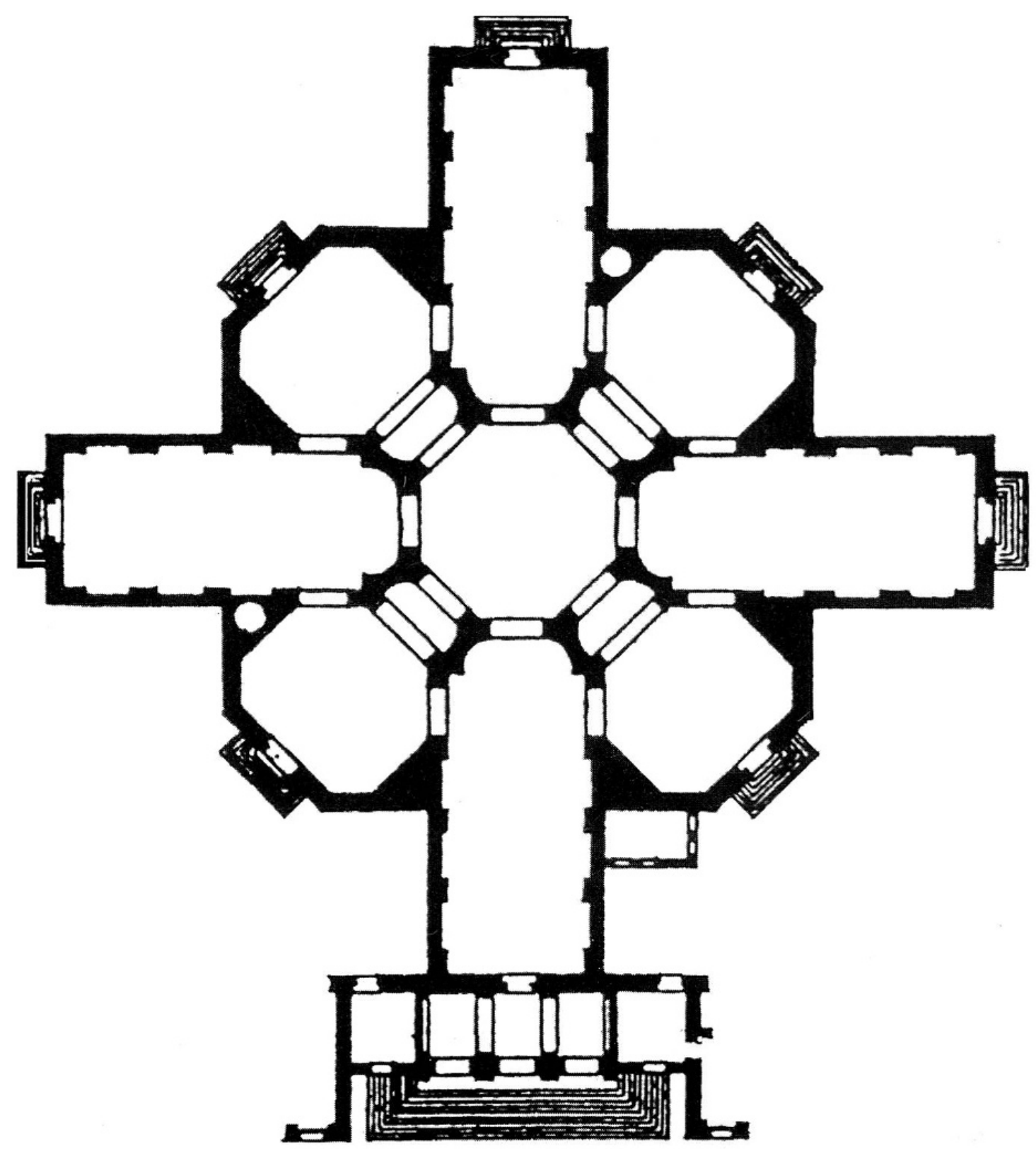

Fig. 2. Plan de l'église St. Louis de la Salpêtrière.

démiaire An IV, par exemple, 26 moururent dans les six mois, et 20 autres dans l'année. ${ }^{4}$

Mourir à la Salpêtrière, c'était aussi la dernière étape pour des malades prononcées incurables par les officiers de santé de l'Hôtel-Dieu. Cet énorme

4 Un sondage du mois d'octobre 1780, 1785, 1790 et Vendémiaire An IV et An IX par exemple, nous donne des admissions de 272,308,183, 207 et 145 femmes (donc en moyenne 223); $5 \%, 5 \%, 10 \%, 5 \%$ et $6 \%$ de folles; à peu près $1 / 4$ d'enfants au-dessous de 12 ans $(27 \%, 22 \%$ et $27 \%$ : les données pour 1780 et 1785 manquent); et les femmes au-dessus de 60 ans montent à $11 \%$ en $1790,48 \%$ en l'An IV et $30 \%$ en 1'An IX. (1Q2 86, 96, 106, 117 et 126). 
hôpital parisien si près de la cathédrale Notre-Dame et enjeambant la Seine était, sous l'ancien régime, ouvert toujours et à tous, donc monstrueusement surchargé de malades (3,500 à la veille de la Révolution). L'Hôtel-Dieu de Paris était anxieux de se défaire de ses incurables. D'où un cortège ininterrompu de brancards et de charrettes portés à bras d'hommes ou trainés par des chevaux vers la Salpêtrière.

Tout au long de ce déversement d'incurables, la police continuait le renfermement des pauvres commencé sous Louis XIV. Parmi les femmes, c'étaient les filles publiques qui constituaient le groupe le plus vulnérable. Le lieutenant de police Jean Charles Pierre Lenoir, par exemple, envoyait des fournées régulières de jeunes femmes en 1780: 66 le 1er juillet, 77 le 4 août, 96 en septembre, 113 en décembre. ${ }^{5}$ Nous savons comment le gouvernement disposait d'elles au début du $18^{\mathrm{e}}$ siècle par l'histoire de Manon Lescaut: comme beaucoup d'autres, elle fut enfermée à la Salpêtrière et puis transportée en Louisiane, parce que la nouvelle colonie française manquait de femmes. Ces cruels transports cessèrent au milieu du dix-huitième siècle, ayant scandalisé le public.

Nous avons vu que, dès le début, l'intention était de moraliser ces femmes par le travail. En principe, toutes les valides travaillaient. Seulement, il n'y avait pas assez d'ouvrage, et les ateliers de couture datent de la fin du Directoire. En fait donc, sous l'ancien régime, une grande partie des vieilles femmes valides enfermées à la Salpêtrière végétaient, oisives.

Une description fiable nous est offerte par le chirurgien Jacques Tenon, dans ses Mémoires sur les hôpitaux de Paris (1788), chef d'œuvre réformateur du siècle des Lumières.

«La Salpêtrière,» écrivit-il, «est le plus grand Hôpital de Paris, et peut-être de l'Europe: cet Hôpital est en même temps une maison de femmes et une maison de force; on y reçoit des femmes et des filles enceintes, des nourrices avec leurs nourrissons; des enfants mâles depuis l'âge de sept à huit mois jusqu'à quatre et cinq ans; de jeunes filles à toute sorte d'âges; de vieilles femmes et de vieux hommes mariés; des folles furieuses, des imbéciles, des épileptiques, des paralytiques, des aveugles, des estropiées, des teigneuses, des incurables de toute espèce, des enfants avec des humeurs froides, etc., etc., etc.» ${ }^{6}$

Un autre observateur des Lumières, le duc de la Rochefoucauld-Liancourt, visita les lieux en 1790, comme président du Comité de mendicité de l'Assemblée constituante. Il était fermement résolu d'apporter aux pauvres de

5 AAPP AHS, Registre des entrées, 1Q2 86. Voir aussi Erica-Marie Benabou, La prostitution et la police des mœurs au $18^{e}$ siècle (Paris: Perrin, 1987), livre remarquable par son érudition, son recours aux sources d'archives et sa compréhension pour les problèmes sociaux responsables pour le commerce de ces femmes.

6 Tenon, Jacques, Mémoires sur les hôpitaux de Paris (Paris: Pierres, 1788), 85. 
France de décents soins de santé. Liancourt nous apprend que la maison de force (dont le bâtiment existe encore sous le vocable de St. Vincent de Paul) comportait quatre secteurs. Le Commun

affreuse demeure [où] sous l'ancien régime, la police de Paris entassait dans une centaine de lits, sans pitié comme sans secours, cinq à six cents filles publiques;

la Prison, pour les femmes qui

par des arrêts ou des ordres particuliers, [c.à.d. des lettres de cachet] étaient condamnées au renfermement;

\section{la Force, où Liancourt comptait 228 femmes dont}

94 sont condamnées pour la vie, elles couchent trois dans un même lit; 134 autres, condamnées à une réclusion plus ou moins longue, la plupart flétries, attendent dans les angoisses la fin de leur châtiment.

On réservait la Grande Force aux prisonnières de marque qui, en fonction du tarif et de leur pension, bénéficiaient d'un régime plus ou moins doux. Liancourt décrit la quatrième section de ce lieu de contrainte, la Correction, comme le

lieu de grande punition pour la maison, [qui] contenait, quand nous l'avons visité, 47 filles, la plupart très jeunes et plus inconsidérées que coupables. Quelques-unes sont des élèves de l'Hôpital et renfermées par les ordres seuls de la supérieure. Des réponses hautaines faites à une officière, des plaintes indiscrètes: faut-il le dire? du vin bu avec des hommes dans un cabaret entretenu dans la maison ... avaient provoqué ces châtiments qui duraient depuis six mois et un an.

«Il est temps,» conclut le duc en 1790, «de reconnaitre et d'enseigner partout qu'une punition qui n'améliore pas est absurde, et que celle qui peut corrompre est criminelle.» ${ }^{7}$

En vue de cette opinion si juste il n'est pas rassurant de trouver dans les «Dispositions générales et provisoires de police, de propreté et de sûreté,» manuscrit qui date du Directoire, l'article 24 de «Police» qui prévoit «une salle de correction pour les perturbateurs et pour les yvrognes.» ${ }^{8} \mathrm{Il}$ est vrai que l'article XIII de l'édit fondateur avait dit textuellement:

Nous donnons tout pouvoir et Autorité de Direction sur tous les pauvres mendiants de Notre Ville et Faubourgs de Paris, tant dehors que dedans ledit Hôpital Général .... Auront pour cet effet les Directeurs poteaux et carcans, prisons et basses fosses dans ledit Hôpital Général et lieux qui en dépendent.

7 Bloch, Camille et Alexandre Tuetey, eds., Procès-verbaux et rapports du Comité de mendicité de la Constituante, 1790-1791 (Paris: Imprimerie nationale, 1911), 625-627.

8 «Dispositions générales et provisoires de police, de propreté et de sûreté,» manuscrit qui [d'après des indications internes] date du Directoire, AAPP, archives anciennes, Fosseyeux 120. 
$\mathrm{Au} 18^{\mathrm{e}}$ siècle, le pouvoir policier de l'institution sur ses pauvres restait formidable. Et alors que la Prison et la Force furent définitivement fermées en 1795, le destin réservé au Commun n'est pas encore clair et il est évident que la quatrième section, la Correction, survécut à la Révolution.

Nous connaissons donc les femmes de la Salpêtrière pour ainsi dire du dehors: leurs logements par les dessins des architectes; leur nourriture et leurs vêtements par les devis de l'économe; la qualité de leur vie par les rapports des réformateurs; leur vie spirituelle par l'aumonier de l'église. Ce sont les représentants du pouvoir qui nous racontent la vie de ces déshéritées; des hommes qui décrivent des femmes. Les femmes elles-mêmes, presque toutes analphabètes, ne nous ont laissé rien d'écrit.

Mais l'on peut tout de même essayer de s'approcher un peu plus de ces femmes à l'aide de registres souvent annotés dont la tenue, ainsi que la teneur nous renseignent de beaucoup de façons. Leur aspect général nous révèle le strict esprit d'ordre qui prévalait dans la bureaucratie de l'Hôpital Général: les listes d'entrées à Bicêtre et à la Salpêtrière sont minutieusement tenues, d'une belle écriture bien lisible sur du bon papier, avec quatre ou cinq individus par page in-folio exactement inventoriés. Pour les prévenus d'un crime, le procès-verbal est transcrit mot-à-mot. On perdait rarement la trace d'un seul de ces milliers de pauvres, ni, semble-t-il, d'un drap ou d'une paire de souliers. ${ }^{9}$ C'est souvent dans la marge d'un registre que la sœur officière ajoutait un commentaire utile pour nous: de fréquents congés de trois semaines et aussi des sorties, toujours consenties et signées par une sœur officière; de fréquentes évasions dûment constatées, en l'An IV, par exemple, elles atteignaient une fréquence étonnante: 68 femmes s'évadèrent pendant les premiers six mois de cette année. ${ }^{10}$ Notre chroniqueur est horrifiée par une évasion avec vol où elle note «évadée, avec la chemise et les habits de la maison, 700 livres à ses camarades, une paire de bas de soie à un particulier, une livre de laine à un autre.» ${ }^{11}$

Sur les pages des registres, la Révolution se traduit souvent en un changement de personnel, donc dans une nouvelle attitude, même dans les détails. On passe d'une écriture où l'on sent un effort laborieux pour former chaque lettre de façon adéquate et lisible, à une main courant avec facilité, qui suit des lignes droites sans qu'on doive les tracer d'avance. On passe de la date du baptême inscrite pour chaque personne (avec la fréquente notation «a apporté son baptistaire,») à la date de naissance, donc du jalon religieux noté

9 Il est vrai qu'un recensement général en 1849 résulte en plusieurs douzaines de femmes qu'on avait perdues de vue, puisque leur registre d'entrée porte la notation «sortie ou décédée.» 10 AAPP, AHS, Registre des sorties, 2Q2, 31.

11 AAPP. AHS. Registre des sorties. 2Q2, An IV, fol.19 r. 


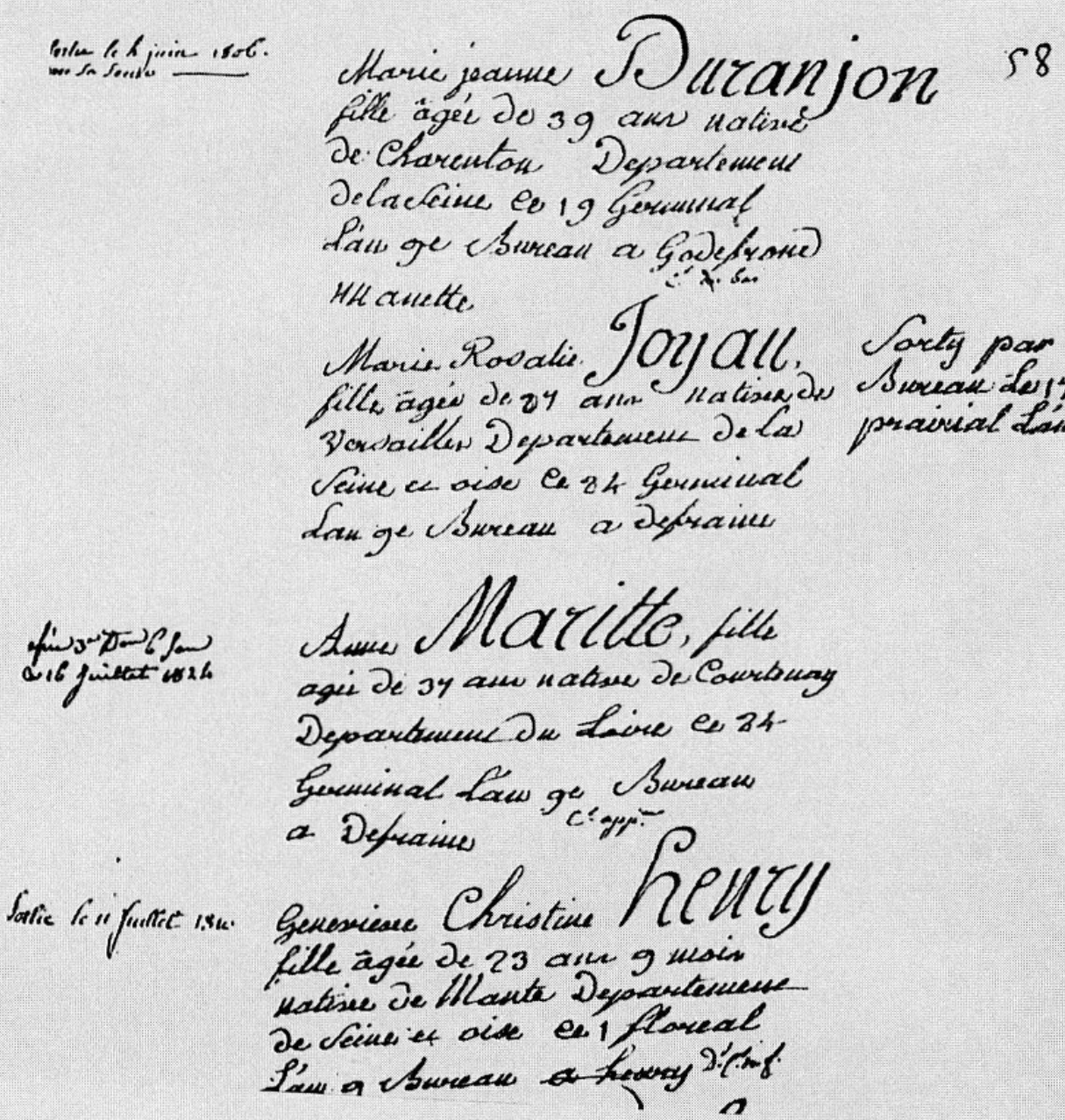

Fig. 3. Archives de l'Assistance publique à Paris. Registres d'entrée, Hospice de la Salpêtrière 1Q2 126, fol. $58 \mathrm{r}$.

par le curé au jalon civil constaté par le maire. Nous ne voyons plus le traditionnel «sortie a fait,» avec la date, mais seul la notation «sortie,» suivie bientôt de l'attestation du médecin. La Salpêtrière passe à un régime civil et médical.

Sous l'empire, quand la police assume un rôle de plus en plus visible dans l'hospice, nous voyons les mentions de professions «lingère, ravaudeuse, dévideuse de soie, couturière, fruitière» céder à des descriptions physiques (plus utiles à Joseph Fouché et ses espions.) Nous lisons, par exemple «cheveux et sourcils chatains, yeux bruns, nez moyen, bouche moyenne, menton rond, visage rond marqué de petite vérole,» détails sans valeur pour un diagnostic médical. ${ }^{12}$ 
Mais l'impression la plus étonnante et permanente que laissent les registres est celle d'un ordre minutieux administratif et bureaucratique en contraste total avec le fourmillement de ces milliers de femmes indigentes en mauvaise santé dont la vie ne valait rien aux yeux de la société. Michel Foucault a donc en partie raison: la société essaya d'imposer un stricte ordre quantitatif à une foule d'êtres humains afin de les intégrer dans son schéma. Mais il a tort de penser que la société tendait à les exploiter et surtout il a tort d'associer les médecins à ce dessein.

Les registres d'entrée nous apprennent qu'au $18^{\mathrm{e}}$ siècle un quart des habitants de la Salpêtrière étaient des enfants, beaucoup d'eux également ramassés par la police, ou parce qu'ils mendiaient, ou parce qu'on les avait vu voler, ou parce qu'ils n'avaient pas de demeure fixe. ${ }^{13}$ Ils étaient «environ seize cents, ${ }^{14}$ enfants de bons pauvres à qui on permettait de vivre en ménage, enfants de prostituées ou de malades nés «dans la maison.» Je pense que leur présence répondait aussi à un instinct maternel aussi profond qu'inavoué de toutes ces officières encore jeunes, renfermées derrière ces hauts murs.

Le duc de La Rochefoucauld-Liancourt était choqué par le manque d'hygiène, commentant qu'«il semblerait presque que l'air, l'eau et la propreté seraient des moyens entièrement inconnus à la Salpêtrière.» Il s'indigna surtout du sort des petites filles:

Si l'on considère ... quelle est la position du bâtiment où sont ces enfants, on le trouve placé près de l'égout de la maison qui répand une odeur infecte dans les grandes pluies. L'amphithéatre d'anatomie est placé au-dessous des dortoirs, et l'air qui entre par les fenêtres est impregné de tous les miasmes putrides qu'exhale la basse-cour où l'on entretient habituellement soixante-quinze cochons mis en pension, au mois, par des charcutiers de Paris. Tous les germes de corruption et de maladies sont rassemblés autour de ces enfants. ${ }^{15}$

On se rend compte des obstacles au changement en lisant la «Réponse de l'économe de la Salpêtrière» à des remonstrances au sujet de la propreté où il argue que

de temps immémorial, les étables à cochons faisant partie de la basse-cour, sont louées à un maître chaircutier [sic] avec la faculté de faire ramasser à son profit dans les cours et dans les baquets placés dans chaque dortoir, les croutes de pain, restes de pois et légumes, lavures et autres choses que les pauvres jettent et qui ne serviraient qu'à faire du gâchis dans la maison.$^{16}$

13 Voir au sujet des enfants perdus dans Paris, par exemple, le récent livre par Arlette Farge et Jacques Revel, Logiques de la foule (Paris: Hachette, 1988).

14 Desgranges, Henri Légier, Hospitaliers d'autrefois: L'Hôpital général de Paris, 1656-1790(Paris: Hachette, 1952), 118.

15 Bloch et Tuetey, Procès-verbaux et rapports, 617.

16 Dommey, économe de la Salpêtrière, Réponse de l'économe de la Salpêtrière au Mémoire sur cet Hôpital lu par M. Cousin, professeur au Collège Royal, dans l'Assemblée des représentants de la Commune de Paris, le 20 juillet 1790 (Paris: Seguy-Thibout, 1790), 8-9. 
Transformer cet hospice en hôpital sera un travail monstre ....qui s'accomplira non par révolution, mais peu à peu.

Ce monde de femmes était régi par vingt-six soeurs officières commandées par une supérieure, petit groupe d'administratrices capables et puissantes dont les historiens n'ont guère fait cas. Leurs Règlements de 1692 établissent en grand détail leurs multiples obligations ainsi que celles de leurs subalternes. Nous intéressent particulièrement les devoirs de la supérieure dans l'infirmerie - qui n'existait jusqu'en 1780 que pour les sœurs, les ecclésiastiques, les employés et les enfants de chœur. Le Règlement assigne à la supérieure le contrôle du travail du médecin, des chirurgiens et de l'administration des remèdes (par. 29). Elle surveille la maison de force et de correction (par. 34) et «se fera rendre compte si les personnes appliquées au service des folles et imbéciles gardent assez de modération dans la conduite dure et sévère qu'elles sont quelquefois obligées d'avoir à leur égard» (par. 38). ${ }^{17}$

Etant données ces lourdes responsabilités, le ton frivole du majeur historien de l'Hôpital général est bien regrettable. Henry Desgranges nous raconte qu'une supérieure était jolie, ou modeste, qu'elle avait un amant et qu'elle allait régulièrement à la messe. Ces bavardages lui semblaient peser plus lourds que de savoir si les pauvres avaient assez à manger.

Et pourtant cette littérature nourrie de petite histoire nous renseigne sur trois aspects importants: d'abord une lutte de tous les jours entre l'économe et les prêtres d'une part, contre la supérieure et ses femmes de l'autre. Dès le début de l'Hôpital général il était convenu que dans cet établissement l'économe devait prendre ses ordres de la supérieure. L'économe se vengeait tant qu'il pouvait. Cette lutte se projetait sur l'arrière-fonds d'une guerre sourde que se faisaient le parti Gallican et Janséniste (soutenu par le Parlement) d'une part, et l'église de Rome de l'autre. Et troisièmement ces relations se compliquaient par suite des réseaux de parenté, d'influences et de faveurs dont semblent avoir dépendu toutes les nominations, toute attribution de fonds, toute décision. Le pouvoir public financier et politique était entre les mains du bureau (c'est à dire des représentants de la haute bourgeoisie) qui se réunissaient le vendredi ..... et puis allaient dîner chez la supérieure. Il semble donc, d'après cette façon d'écrire l'histoire, que le sort des pauvres de la Salpêtrière dépendait de la qualité du dîner et des étincel-

17 Voir le ms \# 70 à la Bibliothèque historique de la Ville de Paris intitulé «Recueil de règlements intérieurs concernant l'hôpital de la Salpêtrière,» surtout le No. 1 «1692. Règlement pour la supérieure des maisons de l'hôpital général,» No. 2 «Règlement pour les soeurs officières de la maison de St. Louis de la Salpêtrière,» et No. 3 «Règlement de ce qui doit être observé chaque jour à la maison de St. Louis de la Salpêtrière.» 
les de la conversation, sinon des relations très personnelles entre les commensaux de ces dîners. ${ }^{18}$

Vue sous l'optique des pauvres et d'observateurs critiques du dehors, cette trentaine de sæurs officières formaient une hierarchie formidable pourvue d'un pouvoir absolu. Bien que créé sous l'influence de Vincent de Paul, ce groupe, avec ses sous-officières et centaines de filles de service, était entièrement laïque. Ces hospitalières portaient un costume voisin de celui des Sœurs de Charité sans partager leurs voeux. Ceci explique que la Révolution les ait épargnées quand elle dispersa les congrégations.

Sous l'ancien régime les officières contrôlaient non seulement la vingtaine d'emplois où habitaient les pauvres, mais aussi l'infirmerie et la prison, la cuisine, la buanderie, la lingerie, l'habillement, la paneterie et les magasins de provisions. Elles géraient aussi un réseau complexe de privilèges et de faveurs régissant l'existence journalière des pauvres. La préférence la mieux justifiée était celle accordée aux «reposantes,» employées de la maison en retraite que l'on y hébergeait pour le reste de leur vie. Elles avaient droit à une chambre seule, au pain blanc et à une chopine de vin. Mais on pouvait aussi payer pour ce privilège: un lit seul dans une petite chambre coûtait 300 livres. ${ }^{19}$ A vrai dire, tout s'achetait à la Salpêtrière qui était après tout un grand village. Un régulier marché avec une vingtaine de boutiques offrait non seulement du pain, des fruits et des saucisses, mais aussi du tabac, du vin et de l'eau de vie. Comme le loyer de ces denières boutiques valait dix fois autant que les autres, on déduit que ce commerce était bien lucratif dans cet hospice de femmes. $^{20}$

Sous le Consulat, le gouvernement essaya d'encourager l'indépendance que semblaient témoigner ces petits marchés et offrait une «pension représentative» de 120 francs par an à toute personne qui se retirerait de l'Hôpital général. Par précaution on ajoutait qu'une place lui resterait réservée. Un cas typique est celui de Jeanne Gérard, 40 ans, qui revient après deux ans et huit mois, ayant renoncé à la pension. Elle a dû se faire embaucher pour travailler puisqu'elle passe reposante de $3^{\text {e }}$ classe. Elle meurt à l'infirmerie générale, à l'âge de 67 ans. ${ }^{21}$ En parcourant les registres, nous rencontrons fréquemment les femmes qui essayaient ainsi de vivre en ville, mais que le manque de famille et de ressources forçait de revenir finir leur vie à l'hospice. Possiblement une longue habitude de vie institutionnelle les avait ren-

18 En principe le bureau était subordonné aux «chefs de la direction» mais ceux-ci n'exerçaient guère leur pouvoir. C'étaient le premier président du Parlement de Paris, le procureur général, l'archevêque de Paris, le prévôt des marchands et le lieutenant général de police.

19 AAPP, AHS, Registre des entrées, 1Q2 117.

20 Voir Weiner, The Citizen-Patient, 67.

21 AAPP, AHS, Registre des entrées, 1Q2 117, folio 12 r. 
dues incapables d'indépendance. Essayer de rallumer le courage de ces déshéritées sera la tâche la plus difficile des réformateurs du monde moderne. Pour le moment, pendant la Révolution, il s'agissait d'abord de l'impérieuse nécessité d'un tri.

A l'admission d'une nouvelle venue, c'est une sœur officière qui décidait dans lequel d'une vingtaine d'emplois la loger: d'après son âge ou ses souffrances chroniques avec les rhumatisantes, les paralytiques ou les épileptiques? d'après son aspect physique avec les mourantes ou bien à l'infirmerie? avec les folles, les prostituées, les privilégiées? Ce choix d'un logis equivalait à un diagnostic quand il s'agissait de femmes malades et, en l'absence d'un médecin sur place, ce sont les officières qui prenaient les décisions.

Nous sommes donc en présence d'une trentaine de femmes énergiques qui fournissaient un travail administratif très dur, prenaient journellement des décisions médicales, imposaient l'obéissance à des centaines d'employés femmes et hommes, et l'ordre à des milliers de femmes épuisées. En histoire de la médecine ce travail reste inapprécié, surtout, je pense, à cause des plaintes bien connues des réformateurs des Lumières qui se heurtèrent au refus de ces officières de modifier leurs traditions. Et pourtant la présence de ce groupe d'infirmières avec une longue expérience allait devenir une ressource précieuse au moment de la transformation de cet hospice en hôpital.

Afin de faire revivre quelques-uns de ces tristes destins, l'historien peut consulter les commentaires individuels notés dans les marges des registres. Commençons par Elisabeth Joseph Pichard de 22 ans qui arrive enceinte d'environ six mois. Elle accouche à la Maternité de Jeanne Edmé trois semaines plus tard. Le bébé meurt, étant sans doute prématuré. Le registre porte seulement que l'état civil de l'enfant était en règle - occurrence commune, sinon fréquente. ${ }^{22}$

Le 10 Vendémiaire An IV on remit au personnel Marie Louise Micque, de quatre ans-et-demi «pour rester à la maison jusqu'à la guérison de sa mère qui est à l'Hospice des Vénériens.» Comme la petite sortit après six semaines, durée du traitement au mercure que dispensait le chirurgien Michel Jean Cullerier, il semble probable que la mère soit venue la chercher. ${ }^{23} \mathrm{Si}$ elle n'était pas revenue, que pouvait faire le personnel sinon garder la petite?

Une autre petite fille était éduquée dans l'école tenue dans l'enceinte de la Salpêtrière; deux institutrices y enseignaient à 24 élèves ce qui traditionnellement faisait partie de l'equipement d'une fille bien élevée: un peu

22 Elisabeth quitte la Salpêtrière le 13 Prairial An IV, donc en mai 1796. AAPP, AHS, Registre des entrées, 1Q2 117, 12 r.

23 Sortie 26 Brumaire IV. AAPP, AHS, 1Q2 117, 6v. 
d'écriture et de calcul, beaucoup de religion et de morale, les soins ménagers. Mais surtout, et c'était cause de scandale, on y élevait les «bijoux,» jolies petites filles sélectionnées par les sœurs pour leur tenir compagnie, ou pour devenir leurs successeurs.

Mais la plupart des enfants échouées à la Salpêtrière manquaient de protection. Abandonnées par des parents connus, ou bien trouvées quelque part par quelqu'un, leur vie s'annonçait dure: Victoire Hiacinte Bivaux, par exemple, de six ans, est «confiée au sieur Duclos, meneur, pour aller à St. Julien du Sault.» Le meneur la remettra à une famille de paysans qui la gardera jusqu'à l'âge de douze ans quand les paiements de l'Hospice des Enfants Trouvés - ridiculement parcimonieux, d'ailleurs - cessent, et elle sera renvoyée à Paris, à l'orphelinat. L'adoption, dont rêvaient les administrateurs parisiens, n'apparaît jamais dans ces registres. La plupart de ces filles échouaient tôt ou tard à la Salpêtrière pour le reste de leur vie..$^{24}$

Le sort de Louise Geneviève Briquet de onze ans «confiée au citoyen Delaître pour aller à l'Epine près Arpajon,» et celui d'une fillette du même âge «envoyée par le juge de paix des Champs Elysées au Cit. Sykes pour aller à St. Rémy,» ${ }^{25}$ était peu enviable. Nous savons qu'à cette aube de la Révolution industrielle des manufactures contractaient avec l'Hôpital général pour leur envoyer un certain nombre de «mains» de cet âge - toujours remplaçables - et que les enfants vivaient dans des conditions déplorables, attachés parfois à leur travail avec boule et chaîne. ${ }^{26}$

Si nous n'avons pas, jusqu'ici, parlé de femmes souffrant de maladies aiguës, c'est que la Salpêtrière faisait fonction d'hospice et non d'hôpital. On transférait les malades: les vénériennes à Bicêtre et, après 1790, à Cochin, les galeuses et teigneuses à St. Louis. Mais c'est surtout vers l'Hôtel-Dieu qu'on les dirigeait. Ces pénibles transports finirent par choquer les contemporains. Le nouveau point de vue suscita la création d'une Infirmerie générale à la Salpêtrière, en 1782, sur l'initiative du ministre des finances Genevois Jacques Necker (1732-1804) et sans doute sur la suggestion de Jean Colombier (17361789) inspecteur général des hôpitaux civils et des maisons de force du royaume. ${ }^{27}$ Le contrôleur Merle nous parle de «ce beau local» dans son «Projet d'un nouveau plan d'organisation» de la Salpêtrière proposé en 1802:

24 AAPP, AHS, Registre des entrées, 1Q2 119 10r.

25 AAPP, AHS, Registre des entrées, $1 \mathrm{Q} 2119,13 \mathrm{v}$ et $15 \mathrm{r}$.

26 Voir Weiner, The Citizen-Patient, 218-219.

27 Necker, Jacques, «Rapport au roi sur l'Hôpital général,» in Euvres complètes, 15 vols. (Paris: Treuttel \& Würtz, 1820), 3, 518-525. 
Les malades ont toute sorte de secours et d'agréments et quand ce ne serait que la confiance justement méritée qu'ont les indigentes envers les médecins et officiers de santé qu'elles sont à portée de voir journellement celà contribuerait beaucoup à leur soulagement. La satisfaction qu'elles éprouvent en voyant les attentions et les soins qu'ils donnent aux malades présagent d'avance aux valides une bien douce consolation dans l'espérance des secours qui les attendent en cas d'indisposition. D'un autre côté, cet établissement sert à former des bons élèves qui ne peuvent que bien profiter sous les yeux de tels maîtres dont le nom de leur chef suffit seulement pour donner les plus grands éloges à la direction de cet établissement. Je ne pense point que l'ordre qui règne à l'infirmerie soit susceptible d'aucun changement. ${ }^{28}$

Avec la création de cette infirmerie cessa le transfer de malades à l'HôtelDieu.

La Salpêtrière pouvait aussi, à la fin du $18^{\mathrm{e}}$ siècle, se décharger vers de nouveaux établissements spécialisés. On pourrait écrire une histoire de la médecine moderne naissante, suivant des bébés syphilitiques à l'Hospice de Vaugirard à partir de 1780; des femmes enceintes à la Maternité de Port Royal à partir de 1796; des jeunes à l'Hôpital des enfants malades à partir de 1802, à l'orphelinat St. Antoine, aux Sourds-Muets de la rue St. Jacques ou bien aux Jeunes Aveugles installés aux Quinze-Vingts. Ce tri déchargeait la Salpêtrière.

En même temps, le Conseil général des hôpitaux et hospices et son $\mathrm{Bu}$ reau central d'admission controlèrent mieux les admissions. On éleva l'âge d'entrée des bons pauvres à 70 ans et triait les malades de son mieux: en 18011802 , par exemple, il n'admit que les $65 \%$ des candidats. Le médecin-en-chef, par contre, continuait à admettre au moins un quart des indigents directement par urgence et le préfet de police transférait régulièrement des femmes arrêtées dans la rue. En conséquence, la population de la Salpêtrière variait entre 4 - et $5,000 .^{29}$

La création d'une infirmerie générale à la Salpêtrière signale un changement fondamental survenu dans les années 1780 . Notons que c'est l'ancien régime qui initia cette révolution en médecine que beaucoup d'interprètes attribuent à tort aux réformateurs révolutionnaires. Ce qui est plus, l'infirmerie nécessita la présence d'un médecin. Jusque là, un solitaire docteur avait été responsable pour l'Hôpital général tout entier. Le soin des pauvres avait été complètement abandonné à quelques chirurgiens secondés, de temps en temps, par un apothicaire. ${ }^{30}$

28 Merle, contrôleur de la Salpêtrière, «Projet d'un nouveau plan d'organisation présenté au conseil général,» Février 1802. Ms, AAPP, Catalogue Fosseyeux, 707-10,31-32.

29 Voir «Règlement pour les admissions dans les hospices, 18 Vendémiaire, An X,» et «Instruction relative à l'admission dans les hospices, 18 Nivôse, An X,» AAPP, B 5089 (7) et (13). Voir aussi Weiner, The Citizen-Patient, chapitre 5, sourtout pp. 146-153 «Triage: The identy of the Outpatient,» et les Tables de l'Appendice C.

30 Quelques historiens nomment comme premier médecin de l'Hôpital général au $18^{\mathrm{e}}$ siècle Raymond Finot, puis Ferme Lhuys l'Epy (1715-1757). Ils auraient logé à la Pitié, tout comme 
Il se trouve que le médecin-en-chef depuis 1795 jusqu'en 1826, Philippe Pinel (1745-1826), se prêta corps et âme à la transformation de la Salpêtrière en «Maison nationale des femmes.» ${ }^{31} \mathrm{Il}$ arriva avec tout le prestige de professeur de pathologie interne à l'Ecole de santé de Paris, et en 1803 il devint membre de l'Académie des sciences. Mais il n'avait pas oublié ses humbles origines. Ses premières activités de médecin-en-chef visèrent l'amélioration de la qualité de vie journalière des femmes dont son poste le rendait responsable. En collaboration avec L. T. Richard d'Aubigny (ca. 1750-1824), membre du Conseil, et le Dr. Benjamin Desportes (17?-1840), membre de la Commission des hôpitaux surveillant la Salpêtrière, Pinel réussit à améliorer la nourriture et à organiser sa distribution au réfectoire (plutôt qu'aux dortoirs), créer des salles de couture pour les femmes valides, veiller à la propreté et créer des promenades salubres, moderniser la buanderie: activités qui peuvent paraître étranges pour un médecin, mais qui haussaient le niveau de vie et le moral des habitants et qui lui gagnèrent la gratitude de ces milliers de femmes. Le fait qu'il habitait la Salpêtrière, auprès de ses malades pendant trente ans sans établir une demeure en ville affermissait leur confiance. ${ }^{32}$

C'est que bien avant la découverte de la «gériatrie» comme spécialité médicale, ${ }^{33}$ Pinel se rendait compte que la vieillesse peut comporter des douleurs, un amoindrissement pénible des capacités visuelles, auditoires, ambulatoires, des pertes de mémoire et de vigueur intellectuelle qui ne sont pas des maladies mais qui apauvrissent l'existence. Ses qualités de gériatre peu-

le chirurgien-en-chef. (Benassis, «La Salpêtrière,» 171.) L'Almanach royal n'indique de médecin de l'Hôpital général qu'à partir de 1782, et de médecin de la Salpêtrière, y demeurant qu'à partir de 1788. Philippe Pinel (1745-1826) arriva le 19 avril 1795, exactement à l'âge de 50 ans. Ses prédecesseurs furent Gaulard, Philip, Jacques Saillant (1747-1804) et Nicolas Chambon de Montaux (1748-1826). D'après l'Almanach, ces médecins ont servi à la Salpêtrière comme suit:

1782-1785 Gaullard

$1786 \quad$ Philip

$1787 \quad$ Philip et Saillant

1788 à $1790 \quad$ Philip et Chambon

$1791 \quad$ Chambon

$1792 \quad$ Philip et Saillant

An II à III Philip.

31 Nom de l'Hospice de la Salpêtrière depuis 1793 jusqu'en 1823 quand on adopta «Vieilesse Femmes.»

32 Pinel décrit ces améliorations récentes dans la Préface à la seconde édition «revue, corrigée et augmentée» de La Médecine clinique rendue plus précise et plus exacte par l'application de l'analyse ou Recueil et résultat d'observations sur les maladies aiguës, faites à la Salpêtrière (Paris: Brosson, 1804) et il les répète, avec quelques additions, dans la troisième édition, 1815.

33 Sur les débuts de la gériatrie, voir, p.e., Ackerknecht, E. H., «Zur Geschichte der Geriatrie,» Schweizerische medizinische Wochenschrift, 1961, 91: 20-21, Butler, R. N., «Geriatric Medicine,» in The Oxford Companion to Medicine, J. Walton, P. B. Beeson, and R. B. Scott, eds. (New York: Oxford University Press, 1986), 2: 469-474, Geller, G., Die Geriatrie an der Sal- 
vent se définir de trois façons: Pinel avait observé que certaines maladies se présentent de façons spéciales dans les personnes âgées et que les vieillards requièrent donc une thérapie propre à leur âge. Il était convaincu que la diminution de certaines forces vitales demandait des adaptations de la part du vieillard plutôt que des interventions médicales. Mais surtout il croyait que vieillir et mourir sont des processus normaux où la fonction du médecin est de réconforter le malade et d'alléger la douleur. On n'a qu'à lire les articles «adynamie,» «asthénie,» «ataxie,» «cachexie,» qu'il contribua au Dictionnaire des sciences médicales en 1812, pour se rendre compte que Pinel fut un pionnier de la gériatrie. ${ }^{34}$ Dans son livre, La médecine clinique, nous lisons:

Un hospice de femmes infirmes, et dont la plupart sont d'un âge très avancé, doit offrir une différence remarquable en le comparant aux autres hôpitaux. ${ }^{35}$

Il pensait que l'air, l'eau, le site de la Salpêtrière compromettaient la santé des vieilles femmes, mais il voyait comme cause principale

... les chagrins qui ont précédé, et une sorte de lutte contre la détresse et l'infortune, l'impression continuée de ces mêmes affections tristes contractées par le séjour de l'hospice, les qualités peu restaurantes de leur nourriture ordinaire, leur état d'isolement et leur séparation de leurs familles, l'idée d'une sorte d'abandon et de réclusion ... ${ }^{36}$

L'histoire typique d'une vielle femme sur le point de mourir dans l'infirmerie générale de la Salpêtrière nous servira d'exemple. Dans l'article «asthénie» Pinel raconte

qu'une femme de quatre-vingt-sept ans avait eu le matin une défaillance de quelques minutes, et les secours du culte lui avaient été administrés; lors de ma visite, elle était revenue à elle-même, et je cherchai à la ranimer par une potion fortifiante et une boisson vineuse; son pouls était devenu naturel, et elle conservait entièrement l'usage de la raison; le soir elle cessa d'exister sans convulsions et sans agonie ..... ${ }^{37}$

Ce qui semble remarquable, c'est que Pinel ou son adjoint restaient au chevet de la malade, l'aidant à mourir. Le médecin-chef acceptait ce triste évènement comme naturel et s'efforçait d'assuager la douleur et la peur. C'est là le vrai «traitement moral.»

pêtrière von Pinel bis Charcot (Zürich: Juris, 1965), Grmek, M. D., On Aging and Old Age: Basic Problems and Historic Aspects of Gerontology and Geriatrics, (The Hague: Junk, 1958), Grmek, M. D., «Les aspects historiques des problèmes fondamentaux de la gérontologie,» XV Congrès International d'Histoire de la Médecine in Archivo Iberoamericano de historia da la medicina y antropologia medica 1957, 9: 245-251, Prus, C. R., (1793-1850), «Recherches sur les maladies de la vieillesse,» Mémoires de l'académie royale de médecine, 1840, 8: $1-27$.

34 Pinel, P., «adynamie,» Dictionnaire des sciences médicales, 60 vols. (Paris: Panckoucke, 1812-1822), 1812,1:161-163 «asthénie,» 1812, 2: 401-406, «ataxie,» 1812, 2: 419-422, «cachexie,» 1812, 3:410-412.

35 Pinel, La médecine clinique, $2^{\mathrm{e}}$ ed., 429.

36 Ibid., 413.

37 Pinel, «asthénie,» 404. 
Pour bien comprendre la transformation de la Salpêtrière au tournant du $19^{\mathrm{e}}$ siècle il convient donc de souligner le rôle de Pinel comme administrateur prenant soin de ses malades, expert en médecine interne et gériatrique, professeur de clinique, initiateur de recherches, sur l'inoculation, sur le pouvoir absorbant de la peau, sur l'usage thérapeutique de l'électricité. Pinel a été mal servi par les historiens qui ne nous montrent que le briseur des chaînes des aliénés. Je pense que qu'il faut plutôt voir en lui un médecin qui traitait les malades pauvres avec un respect largement absent jusque-là, s'intéressant autant à leurs maladies physiques que mentales. Ainsi vu, il devient un fondateur d'une médecine publique compréhensive, démocratique, psychologique et moderne. Néanmoins Pinel reste le fondateur de la psychiatrie en France.

En 1802, le gouvernement décida de transférer le traitement de toutes les femmes malades de l'esprit de la région parisienne à la Salpêtrière. Une des principales raisons est que le ministre de l'intérieur Jean Antoine Chaptal (1756-1832) avait, comme tout le monde, lu un livre novateur paru en 1800: le Traité médico-philosophique de l'aliénation mentale ou la manie de Philippe Pinel. Chaptal avait, comme tant d'autres, appris à considérer les folles comme des malades et non des criminelles et, en tant que médecin, il croyait volontiers ce que professait son ami Pinel, que les fous, et surtout les maniaques, étaient susceptibles de guérison. Mais il fallait des preuves.

Le 15 Germinal An X, Pinel et son adjoint Augustin Jacob Landré-Beauvais (1772-1840) commencèrent à visiter systématiquement toutes les femmes atteintes de maladies mentales hospitalisées à la Salpêtrière. Leur but était de sélectionner celles susceptibles d'un traitement.

Bien entendu, le travail des deux aliénistes ne devait pas se limiter à un tri retrospectif. Ils examinèrent également toutes les femmes amenées à la

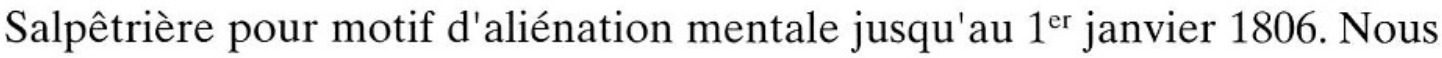
connaissons le raisonnement, le projet et les résultats de ce travail par trois mémoires présentés par Pinel à l'Académie des sciences et à la Société médicale d'émulation. ${ }^{38}$

38 Pinel, Philippe, «Recherches sur le traitement général des femmes aliénées dans un grand hospice, et résulats obtenus à la Salpêtrière après trois années d'expérience,» Le Moniteur universel, 11 Messidor An XIII, 1158-1160, publié dans Gauchet, Marcel et Gladys Swain, Des passions considérées comme causes, symptômes et moyens curatifs de l'aliénation mentale, par E. Esquirol (Paris: Librairie des deux mondes, 1980), 104-113, Pinel, Philippe, «Résultats d'observations et construction de tables pour servir à déterminer le degré de probabilité de la guérison des aliénés, Mémoires de la classe des sciences mathématiques et physiques, Institut national de France, lère série, 1807, 8: 169-205, Pinel, Philippe, «Résultats d'observations pour servir de base aux rapports jurdiques dans les cas d'aliénation mentale,» Mémoires de la société médicale d'émulation, 1817, 8: 675-684. 
L'expérience avec ces malades confirma par des centaines d'exemples les théories et les pratiques annoncées dans les écrits préalables de Pinel tels que le «Mémoire sur la manie» de $1794^{39}$ et le Traité de 1800. Beaucoup de ces malades étaient curables, surtout celles entre 20 et 40 ans. Une prompte intervention du médecin aliéniste donnait les meilleurs résultats. Surtout, il fallait éloigner la malade de sa famille, l'isolant ainsi de la cause probable de son aliénation. Le plus souvent, une seule faculté mentale était atteinte d'aliénation: une fois reconnue, cette condition indiquait le traitement. Il devait se fonder sur une confiance totale de la malade en son médecin qui usait de son pouvoir pour ramener la malade à elle-même. Pinel s'intéressait beaucoup aux rechutes, à la maladie qu'il appelait manie périodique. Il proposait une théorie thérapeutique originale, visant à ce que le médecin se rapproche de la malade pendant les intervals lucides afin de gagner sa confiance, et la faire collaborer à son propre rétablissement. ${ }^{40}$

C'est la première fois que les malades de l'esprit d'un grand hôpital public français aient vu le médecin prendre le temps de se pencher sur leur sort individuel. Peu à peu elles lui ont fait confiance, elles ont répondu à ses questions, surtout le voyant revenir plusieurs fois leur parler, et elles ont fini par révéler des détails personnels, parfois intimes de souvenirs ou d'idées qui les troublaient. Souvent un membre de la famille a dû fournir des informations supplémentaires, surtout sur ce que Pinel appelait les causes occasionnelles, c.à.d. les évènements qui avaient précipité la maladie. Comme il s'agissait de femmes, il est évident que des problèmes d'accouchement, de menstruation, de ménopause prévalaient.

Mais Pinel s'est aussi rendu compte - et c'était bien douloureux pour lui - que l'attention continue accordée par le médecin à chaque malade était une chimère tant qu'il n'y avait que deux médecins pour près de mille aliénées, sans parler d'une infirmerie de 300 à 400 patientes. Pinel n'a pu que montrer le chemin.

Les changements à la Salpêtrière - la création de l'infirmerie générale, le début de recherches, de médecine gériatrique, d'enseignement clinique au lit du malade et de traitement de l'aliénation mentale, font partie d'une évolu-

39 Le texte français a été publié par Jacques Postel dans Genèse de la psychiatrie: Les premiers écrits de Philippe Pinel (Paris: Le sycomore, 1981), 233-248. Pour la présentation de ce mémoire, voir Dora B. Weiner, «Philippe Pinel's 'Memoir on Madness' of 11 December 1794: A Fundamental Text of Modern Psychiatry,» American Journal of Psychiatry, 1992, 149: $725-732$.

40 Voir, à ce sujet, la thèse de Gladys Swain, Le sujet de la folie: Naissance de la psychiatrie (Toulouse: Privat, 1977). 
tion fondamentale de cet hospice en hôpital - ce que mes collègues historiens de la médecine aiment appeler «médicalisation.» Cette transformation commence vers la fin des Lumières, mais les dernières traces de l'hospice n'ont disparues qu'en $1970 .{ }^{41}$ Elle résulte d'un changement du regard que la société porte sur l'indigent malade. Comme l'a dit si bien Marcel Candille, on transforma les pauvres malades en malades pauvres.

Le rôle spécial des femmes s'atténuait à mesure que la Salpêtrière devenait au $19^{\mathrm{e}}$ siècle un hôpital normal. Elle eut un long moment de gloire quand, deux générations après les changements fondamentaux que Pinel introduisit dans la vie et le traitement de ces femmes, leurs petites filles révélèrent à Jean Martin Charcot les secrets de l'hystéro-épilepsie. Ce serait un autre sujet, pour une autre conférence, que d'analyser le rôle de ces femmes dans l'œuvre de Charcot.

L'autre aspect exceptionnel, du point de vue de la femme, est la fondation, sous la $3^{\text {e }}$ république, de la première école d'infirmières en France. On sait que depuis huit cents ans, depuis les croisades, soigner les malades correspondait à une vocation, et que les ordres religieux, masculins mais surtout féminins, qui se vouaient à cet état, représentent une création originale du monde catholique occidental. A mesure que le régime politique en France devenait plus laïque, on désirait voir des infirmières séculières. D'où l'importation de «rossignols anglais,» English nightingales, et, en 1907, la création de l'école d'infirmières de l'Assistance Publique à la Salpêtrière. ${ }^{42}$ En dépit de leur grande compétence scientifique, la situation professionnelle de ces femmes modernes révèle un contraste surprenant: c'est que le pouvoir quasi absolu des sæurs officières d'antan fut aboli en 1790 par la Révolution. Depuis ce moment, ce sont les hommes qui commandent, dans l'administration de la Salpêtrière aussi bien que dans ses services médicaux.

Cette observation donne à réfléchir au sujet d'une remarque de Jacques Tenon qui savait qu'il faut faire évoluer l'hôpital suivant les besoins de la société.

«Les hôpitaux,» écrivit Tenon en 1788, «sont, en quelque sorte, la mesure de la civilisation d'un peuple. Ils sont plus appropriés à ses besoins et mieux tenus, à proportion de ce qu'il est plus rassemblé, plus humain et plus instruit. ${ }^{43}$

41 Greffe, Florence, «La Salpêtrière, dans la première moitié du 19ème siècle,» La Salpêtrière hier et aujourd'hui, Numéro spécial de L'Hôpital à Paris, Août 1982, 43-53 Voir p. 51; voir aussi Berenblit, Eliane, «Approche de la psychiatrie à travers l'histoire à la Salpêtrière,» Certificat d'infirmière, Lycée Rabelais, Octobre 1980 (AAPP) D 691.

42 Croix-rouge française, Histoire des infirmières, 2 vols. (Paris:France-Sélection, 1988. Voir aussi Weiner, Dora B., «The French Revolution, Napoleon and the Nursing Professions,» Bulletin of the History of Medicine, 1972, 46:274-305.

43 Tenon, Mémoires, 1. 
Tenon aurait été content d'apprendre que la Salpêtrière, qui avait manqué à sa mission sous l'ancien régime, s'est peu à peu transformée en ce qu'elle devait être. Appliquée aux femmes, cette fameuse citation évoque les abus de l'ancien régime ainsi qu'une évolution souhaitable dans l'avenir.

Ce texte de la conférence annuelle Guggenheim-Schnurr fut présenté le 8 octobre 1994 pendant la réunion anuelle de la Société suisse d'histoire de la médecine et des sciences naturelles à Aarau. L'auteur tient à remercier ses hôtes, et surtout le professeur Jean Jacques Dreifuss, président sortant et le professeur Urs Boschung, président-élu et les autres organisateurs de leur bienveillante hospitalité.

Les recherches pour ce travail ont été entreprises avec l'aide d'une bourse de la Fondation Culpeper à laquelle je tiens à exprimer ici ma reconnaissance. Ces recherches, poursuivies surtout aux Archives de l'Assistance Publique à Paris, n'auraient pu aboutir sans le soutien bienveillant de Mme Valérie Poinsotte, conservateur, et la collaboration de Mme Martine Bui, archiviste. Qu'elles reçoivent ici l'expression de ma reconnaissance.

\section{Note bibliographique}

Sur l'histoire générale de la Salpêtrière, consulter Benassis, Dr. «Hospice de la Salpêtrière,» in «Promenades médicales,» Revue thérapeutique, 1936,4:104-113,136-144, 168-177, 199-208, Boucher, Louis, La Salpêtrière, son histoire de 1656 à 1790. Ses origines et son fonctionnement au 18ème siècle (Paris: Progrès médical,1883), Couteaux, Jean, «L'Histoire de la Salpêtrière,» Revue hospitalière de France, 1944, 9: 106-127, 215-242, Gasco, R., La Salpêtrière, 1789-1794, (Paris: Diplôme d'études supérieures d'histoire, M. Reinhard, dir., 1969), Guillain, G. et Mathieu, P., La Salpêtrière (Paris: Masson,1925), Henry, Marthe, La Salpêtrière sous l'ancien régime: Les origines de l'élimination des antisociaux et de l'assistance aux aliénés chroniques (Paris: Thèse médecine, 1922), Imbert, Jean, «L'Hôpital général et la Salpêtrière aux 17ème et 18ème siècles,» Société internationale d'histoire de la psychiatrie et de la psychanalyse, Deuxième colloque (Frénésie éditions, 1987), 9-18, Larguier,Leo, La Salpêtrière, Série: Les vieux hôpitaux français (Lyon: Ciba, 1939), Prévost, abbé, Histoire de Manon Lescaut et du Chevalier Des Grieux (Paris: Michel Lévy, 1860), Quétel, Claude, «La question des insensés à l'Hôpital général de Paris à la fin du 18ème siècle,» Société internationale, Deuxième colloque, 27-39, Sainte Fare Garnot, Nicolas, «La folie au grand siècle, l'exemple de la Salpêtrière, 1656-1703), Ibid., 19-26. La Salpêtrière hier et aujourd 'hui, Numéro spécial hors série de L'Hôpital à Paris, août 1982, Simon, Nadine et Jean Franchi, La Pitié-Salpêtrière (Paris: Edition de l'Arbre à Images, 1986), Tesson, M., secrétaire de la Commission municipale du Vieux Paris, «Visite de la Salpêtrière,» Commission municipale du Vieux Paris: Procès-verbaux, 1903, 182-195.

Les articles suivants offrent des perspectives intéressantes: Berenblit, Eliane, «Approche de la psychiatrie à travers l'histoire à la Salpêtrière,» Certificat d'infirmière, Lycée Rabelais, Octobre 1980 (AAPP) D 691, Hautecœur, Louis, «L'Architecture hospitalière et la Salpêtrière,» Médecine de France, 1958, 96: 21-36. 
Pour une appréciation générale de la Salpêtrière dans la vie médicale de Paris, voir Du Camp, M., Paris, ses organes, ses fonctions et sa vie dans la 2nde moitié du 19ème siècle, 6 vols. (Paris: Hachette, 1875), 2 ed ed, 3: 322-329, 4: 52-60, 5: chaps 26 et 27, Guillot,A.,Paris qui souffre: Les prisons de Paris et les prisonniers (Paris:Dentu,1890), et Weiner, Dora B., The Citizen-Patient in Revolutionary and Imperial Paris (Baltimore, MD: The Johns Hopkins University Press, 1993), Part III: Institutions: The Citizen-Patient and the Hospital.

Les archives suivantes ont été consultées:

Archives de l'Assistance publique à Paris. Archives hospitalières. Salpêtrière, dorénavant AAPP AHS:

1K 1-2 Registre d'inscription des employés et gens de service

2K 1-7 Registre des appointements 1814-1820

P 5 Journal de l'économat: dépenses, An VIII-An XIII

1Q2 86 (1780) à 141 (1820) Registres des entrées

2Q2 23 (1789) à 31 (An IV-V) Registres des sorties

6Q1 Registre d'entrée des aliénées volontaires

6Q2 Registre d'entrée des aliénées d'office

6Q3 9-9 bis Registre d'admission des folles

6Q6 2 Journal général: Bijoux déposés par les aliénées, 1807-1847

7Q 1 Registre des femmes enceintes, 1793- 24 Thermidor V

7Q 18 Registre des teigneux, 1793-An IX

7Q 31 Registre des décès de militaires, 1814-1815

6R 1 Registre d'observations médicales, 1795-1853, $5^{\mathrm{e}}$ division, $1^{\mathrm{e}}$ et $2^{\mathrm{e}}$ sections

6R 21 Archives médicales, aliénés, 1815-1851 